\title{
MiR-16 Induced the Suppression of Cell Apoptosis While Promote Proliferation in Esophageal Squamous Cell Carcinoma
}

\author{
Yi Zhu ${ }^{a, b}$ Yang Xia ${ }^{a}$ Hongyan Niuc Yijiang Chen ${ }^{a}$ \\ aDepartment of Thoracic Surgery, The First Affiliated Hospital of Nanjing Medical University, Nanjing, \\ 'Department of Thoracic Surgery, Huai'an Hospital Affiliated of Xuzhou Medical College and Huai'an \\ Second People's Hospital, 'Department of Clinical Laboratory, Huai'an Hospital Affiliated of Xuzhou \\ Medical College and Huai'an Second People's Hospital, Huai'an, China
}

\section{Key Words}

MicroRNA • Apoptosis $• 3^{\prime} U T R \cdot R E C K \cdot S O X 6$

\begin{abstract}
Background/Aims: MicroRNAs (miRNAs) are non-coding small RNAs that regulate cell proliferation and functions by interfering with the translation of target mRNAs. Altered expression of miRNA is known to induce various human malignancies, but little is known about the role of miRNAs in esophageal squamous cell carcinoma (ESCC). Methods: RT-PCR and Western blot were used to examine the expression of miRNAs and candidate genes in 40 pairs of squamous cell carcinoma of human. MiR-16 mimics and inhibitor were transfected in human TE-1 and Eca-109 cells before detecting the cell migration, proliferation, apoptosis and cell cycle. The regulation mechanism was confirmed by luciferase reporter assay. Caspase- 3 and 9 were detected by RT-PCR and Western blot. Results: Aberrant increased level of miR-16 was detected in the ESCC tissues compared with the corresponding adjacent tumor tissues. MiR-16 could inhibit cell apoptosis while promote cell proliferation by down-regulating RECK and SOX6 in TE-1 and Eca-109 cell lines through binding the 3'UTR of both RECK and SOX6 mRNA. Conclusions: Aberrant expression level of miR-16 could suppress cell apoptosis while promote growth by regulating RECK and SOX 6 which play important roles in the pathogenesis of ESCC.
\end{abstract}




\section{Introduction}

Esophageal carcinoma is one of the most deadly malignant tumors in the world. It has become the sixth leading cause of death and the eighth most frequently diagnosed cancer [1]. Esophageal carcinoma is divided into three types including squamous carcinoma (ESCC); adenocarcinoma and undifferentiated carcinoma according to the results of pathological diagnosis [2]. ESCC is the most common subtype with a poor treatment and survive rate with a 5-year overall survival rate about $14 \%$ [3]. Traditional treatment on ESCC including chemotherapy, radiation therapy and esophageal resection is not enough comparing with the increasing number of people suffering with ESCC. Development a new diagnosis method and a novel treatment are very necessary.

A large amount of work has been conducted on studying the mechanism of the pathogenesis of ESCC. Researchers have found that a large number of aberrant expression level of genes participant in the progression of ESCC. The interaction between these genes mostly exists in the transcription level and post-transcription level. MicroRNAs are defined as one of the most important member in the post-transcription level regulation. MiR-375 have been proved to inhibit tumor growth and metastasis in ESCC by down-regulating the IGF1R, acting as a strong tumor-suppression factor [4]. Besides, miR-138 could suppress the level of NF- $\kappa$ B which promotes lipid raft formation in ESCC [5]. All these evidence indicate that miRNAs play an important role in the progression of ESCC.

MiRNAs are small, non-coding RNA molecules of 19-25 nucleotides which have been reported to play important roles by regulating cell differentiation, proliferation, migration and apoptosis. MiRNAs negatively regulate their target genes expression at the posttranscription level through binding to 3' untranslated regions (UTRs) of their targets message RNAs [6]. To date, more than 800 miRNAs have been identified in mammalian cells. Many of them have also been implicated in cancer development and metastasis [7].

Based on the results of the aberrant expression level of miRNAs in the research published, we found that miR-16 was over-expressed in the tumor tissues compared with the adjacent corresponding tissues and in ESCC cell lines compared to normal squamous epithelial cell lines which indicated miR-16 may involve in the pathogenesis of ESCC [8, 9]. Besides, miR16 has been identified to participate in multiple pathways by regulating different genes in various cancers, such as non-small cell lung cancer; ovarian cancer; bladder cancer, however, in which pathway of miR-16 participate still remains unknown[10-12].

\section{Materials and Methods}

\section{Patient and Tissue Samples}

40 patients with ESCC had undergone routine surgery at The First Affiliated Hospital of Nanjing Medical University, Nanjing City, Jiangsu Province, China from May 2012 and November 2012. ESCC samples and the adjacent ESCC tissues taken from the 60 patients were collected, immediately snap frozen in liquid nitrogen, and stored at $-80^{\circ} \mathrm{C}$ until RNA extraction. The tumors were classified according to World Health Organization classification. This study was approved by the Ethical Committee of The First Affiliated Hospital of Nanjing Medical University, and every patient had written informed consent.

\section{Cell Culture and Transfection}

ESCC cell lines and TE-1 and Eca-109 were purchased from the Chinese Academy of Sciences Cell Bank. All cells were cultured in RPMI-1640 (Gibco, USA) supplemented with $10 \%$ fetal bovine serum (Invitrogen, Carlsbad, USA) and grown in humidified $5 \% \mathrm{CO} 2$ at $37^{\circ} \mathrm{C}$. MiR-16 mimics and normal control were obtained from Genepharma (Shanghai, China).The transfection was conducted by using Lipofectamine 2000 (Invitrogen Corp, CA, USA). 


\begin{tabular}{|c|c|c|}
\hline Cellular Physiolosy & Cell Physiol Biochem 2014;33:1340-1348 & \\
\hline and Biochemistry & $\begin{array}{l}\text { Dol: 10.1159/000358701 } \\
\text { Publisned onine: Wvay 05, } 2014\end{array}$ & $\begin{array}{l}\text { O } 2014 \text { S. Karger AG, Basel } \\
\text { www.karger.com/cpb }\end{array}$ \\
\hline
\end{tabular}

Quantitative RT-PCR

Quantitative real time polymerase chain reaction (qRT-PCR) was performed to determine the expression levels of miR-16 and mRNAs of all related genes. Total RNA was obtained from tissues using TRIzol reagent as described by the manufacturer (Invitrogen Life Technologies Co, CA, USA). For mRNA detection, total RNAs (500 ng) were reverse transcribed using the reverse transcription kit (Takara, Tokyo, Japan). $\beta$-actin was used as an internal control. TaqMan ${ }^{\circledR}$ MicroRNA Assays (Applied Biosystems, CA, USA) was used as the probe for has-miR-16 and has-U6 which act as a normalized control. QRT-PCR was performed using ABI Prism 7900HT (Applied Biosystems, CA, USA) according to the direction of the reagents

\section{Protein analysis}

For western-blotting, total proteins were extracted from tissues or cultured cells using RIPA buffer containing PMSF (Roche, Basel, Switzerland). Equal amount of proteins (100ug) were separated with $7.5 \% / 12.5 \%$ sodium dodecyl sulphate polyacrylamide gel electrophoresis (SDS-PAGE) and transferred to polyvinylidene fluoride (PVDF) membrane. The blots were developed using ECL reagent (Millpore, MASS, USA). Equal amount of protein loading in each lane was confirmed using GAPDH antibody.

\section{Cell proliferation assays}

3-[4, 5-dimethylthiazol-2-yl]-2, 5-diphenyltetrazolium bromide (MTT; Roche, Molecular Biochemicals, Mannheim, Germany) assay was used to examine the cell proliferation. Absorbance at $490 \mathrm{~nm}$ was measured by the TECAN infinite M200 Multimode microplate reader (Tecan, Mechelen, Belgium). Each assay was performed in triplicate and repeated 3 times independently.

\section{Cell cycle and apoptosis analysis}

Cells were transfected with miR-16 mimics as well for $48 \mathrm{~h}$. All experiments were analyzed by BD Biasciences FACS Calibur Flow Cytometry (BD Biasciences, NJ, USA). The tests were repeated for three times with triplicate per experiment.

\section{Dual-luciferase reporter assay}

The 3'-UTR sequence of RECK and SOX6 predicted to interact with miR-16 or a mutated sequence with the predicted target sites were inserted into the KpnI and SacI sites of pGL3 promoter vector (Genscript, Nanjing, China). For reporter assay, cells were plated onto 24-well plates and transfected with $100 \mathrm{ng}$ of pGL3-RECK, pGL3-SOX6 or pGL3-RECK-mut, pGL3-SOX6-mut and miR-16 mimic, respectively by using Lipofectamine 2000 (Invitrogen Corp, CA, USA). A Renilla luciferase vector pRL-SV40 (5 ng) was also cotransfected to normalize the differences in transfection efficiency. Transfection was repeated three times in triplicate

\section{Statistical analysis}

The method of 2- $\triangle \mathrm{Ct}$ was used to analyze the results of RT-PCR in all the experiments performed in this study. Statistical analysis was performed using STATA 9.2, and presented with Graph PAD prism software. Experimental data of tissue samples are presented as box plot of the median and range of log-transformed relative expression level which was analyzed by Wilcoxon rank-sum (Mann-Whitney) test. While the results obtained from experiment in vitro assays are presented as mean \pm SE from three separate experiments in triplicates per experiment, and the data was analyzed by double-sided Student's t-test. Results were considered statistically significant at $\mathrm{P}<0.05$.

\section{Results}

\section{MiR-16 was increased in human ESCC tissues}

First, we analyzed the expression levels of miR-16 in both ESCC tissues and adjacent tissues by qRT-PCR. Significantly, we found that miR-16 expression was increased in ESCC tissues ( $n=40$, Fig. 1A). A positive correlation of miR-16 between the tumor tissues and adjacent tumor tissues was obtained which indicated that miR-16 was acted as an oncogene (Fig. 1B). After analyzing the clinical information of patients, we found that the aberrant 


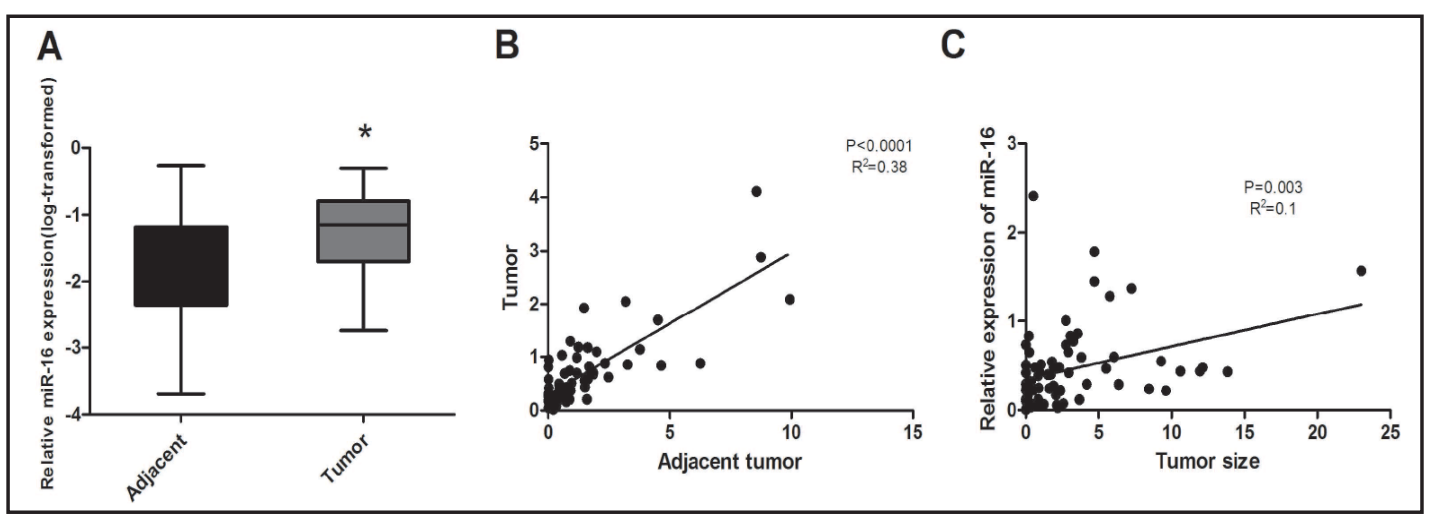

Fig. 1. A: The expression levels of miR-16 in human ESCC tissues and adjacent corresponding tissues $(n=40)$ were evaluated by qRT-PCR. B: A positive correlation of miR-16 between the tumor tissues and adjacent tumor tissues. C: A positive correlation was obtained which indicated that the expression level of miR-16 was highly associated with tumor size in ESCC. * indicates significant difference $(\mathrm{P}<0.05)$.

Table 1. The clinicopathological relevance analysis of miR-16 expression in ESCC patients

\begin{tabular}{|c|c|c|c|}
\hline \multirow[b]{2}{*}{ Feather } & \multicolumn{2}{|c|}{ miR-16 } & \multirow[b]{2}{*}{$P$ value } \\
\hline & Low & High & \\
\hline All cases & 20 & 20 & \\
\hline \multicolumn{4}{|l|}{ Age } \\
\hline$<60$ & 15 & 11 & 0.185 \\
\hline$\geq 60$ & 5 & 9 & \\
\hline Gender & & & 0.677 \\
\hline Male & 16 & 17 & \\
\hline Female & 4 & 3 & \\
\hline Tumor Size $(\mathrm{cm})$ & & & 0.006 \\
\hline$\leq 5 \mathrm{~cm}$ & 3 & 16 & \\
\hline$>5 \mathrm{~cm}$ & 17 & 4 & \\
\hline Tumor Location & & & 0.633 \\
\hline Middle & 3 & 2 & \\
\hline Lower & 17 & 18 & \\
\hline Tumor Capsular & & & 0.151 \\
\hline Incomplete & 4 & 1 & \\
\hline Complete & 16 & 19 & \\
\hline TNM stage(I:II:III) & $9: 6: 5$ & $2: 3: 15$ & 0.032 \\
\hline
\end{tabular}

Fig. 2. Flow cytometry assay was performed to assess cell apoptosis. Cells were tranfected with miR-16 and control. The apoptosis of both TE-1 and Eca-109 were inhibited when cells treated with miR-16 mimics. The number of apoptosis cells was presented with Mean \pm SE. * indicates significant difference compared with that of control cells $(\mathrm{P}<0.05)$.
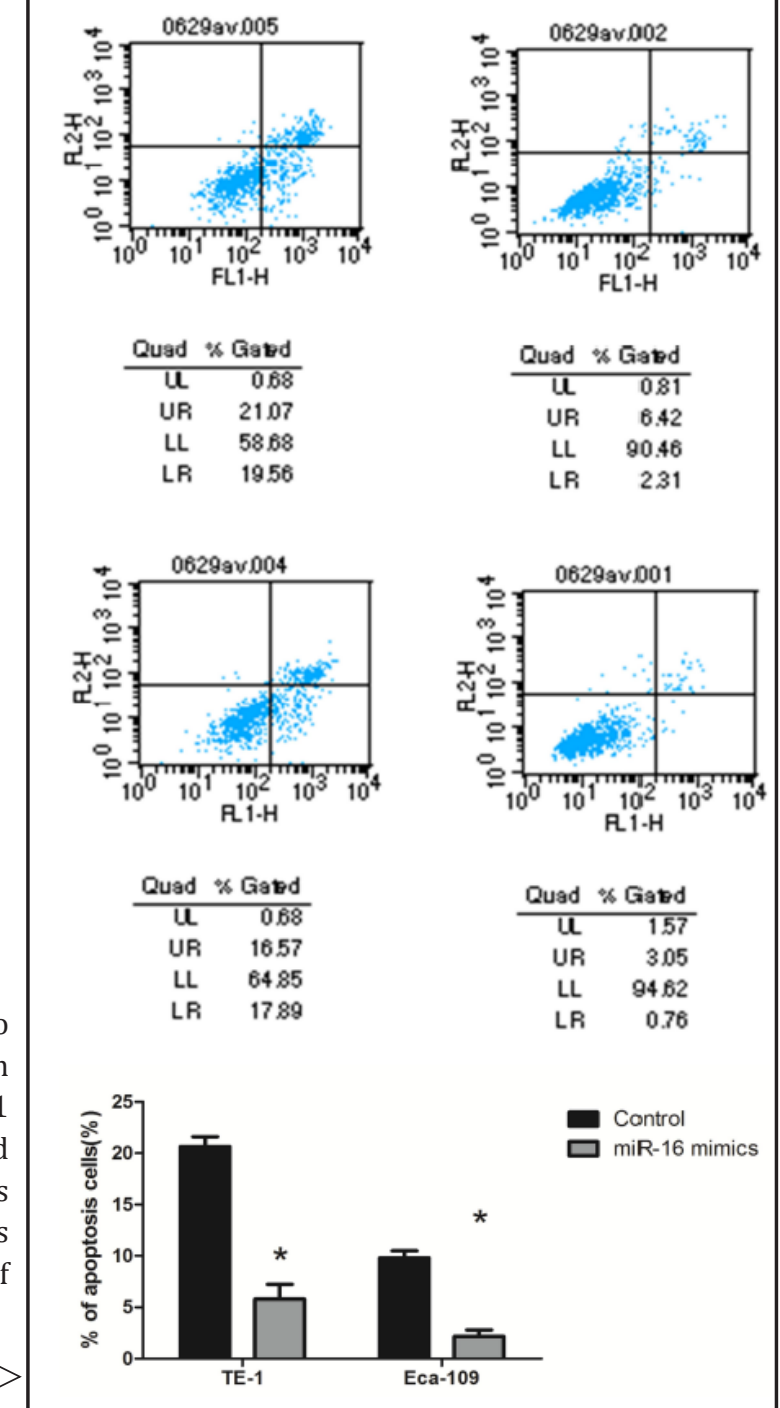

expression level of miR-16 was highly associated with the tumor size of patients (Fig. 1C), indicating that miR-16 may play an important role the pathogenesis of ESCC (Table 1). 


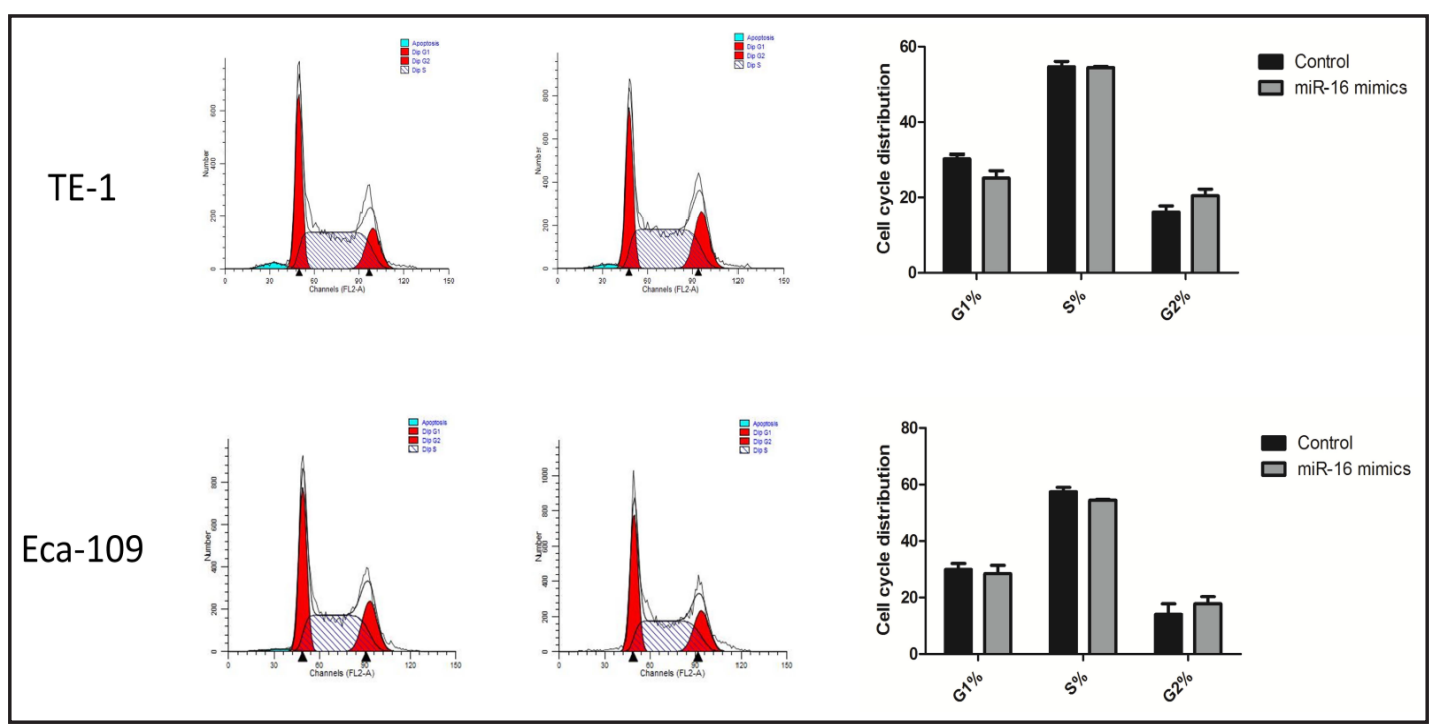

Fig. 3. Flow cytometry assay was performed to assess cell cycle. Cells were tranfected with miR-16 and control. The cell cycle of both TE-1 and Eca-109 were no difference between cells treated with miR-16 mimics and control. The distribution of cell number was presented with Mean \pm SE.

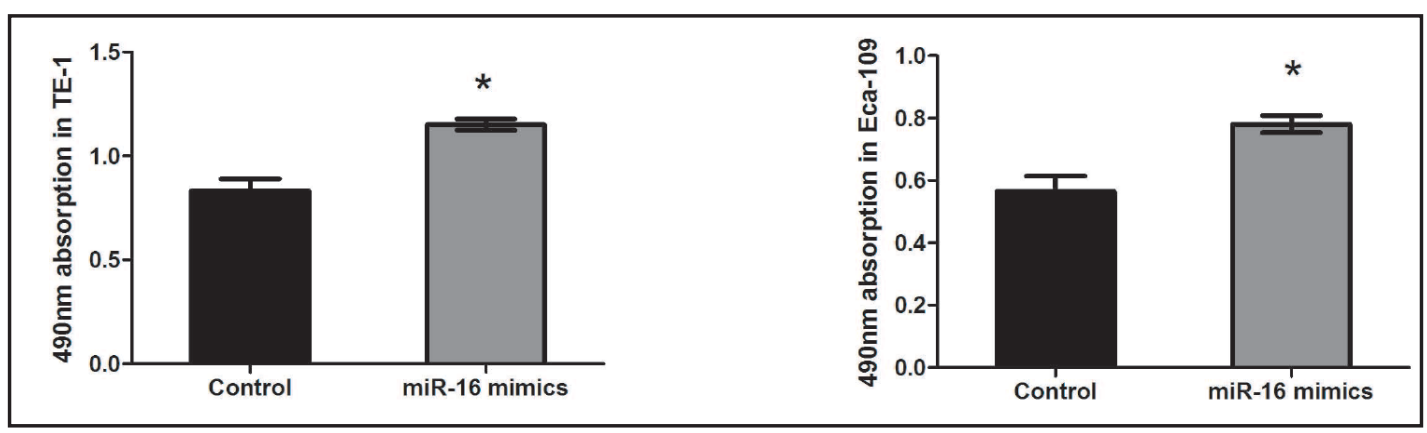

Fig. 4. MTT was conducted to investigate the proliferation of cells treated with miR-16 mimics and control in TE-1 and Eca-109 cell lines. The Absorbance at $490 \mathrm{~nm}$ was presented with Mean \pm SE. * indicates significant difference compared with control group $\mathrm{P}<0.05$.

Therefore, based on this expression pattern, we chose the TE-1 and Eca-109 cell lines to determine the functions of miR-16 in ESCC.

MiR-16 inhibited cell apoptosis while promote cell growth without affecting cell cycle

To detect the functional roles of miR-16, we then examined the effect of miR-16 on cell proliferation, cell apoptosis and cell cycle. A suppressive effect was observed in cell apoptosis in both TE-1 and Eca-109 cell lines by using flow cytometry analysis (Fig. 2). In addition, we used flow cytometry analysis to investigate whether miR-16 was involved in cell cycle progression. We found that the miR-16 mimics did not affect cell cycle progression when compared with the control (Fig. 3). Besides, the results of MTT assay indicated that the absorption in $490 \mathrm{~nm}$ of cells was statistically different between cells transfected with miR-16 and the control (Fig. 4). Furthermore, in order to detect the inhibition affection of cell apoptosis, we detected the viability of Caspase 3 and 9 in cells transfected with miR16 mimics and control to explore the correlation between miR-16 and apoptosis. The results indicated that both the cleaved Caspase 3 and 9 was decreased in cells transfected with miR-16 mimics comparing with the cells transfected with control (Fig. 5). This result suggested that the aberrant decreasing level of miR-16 may have the ability of suppressing cell apoptosis in ESCC cell lines. 


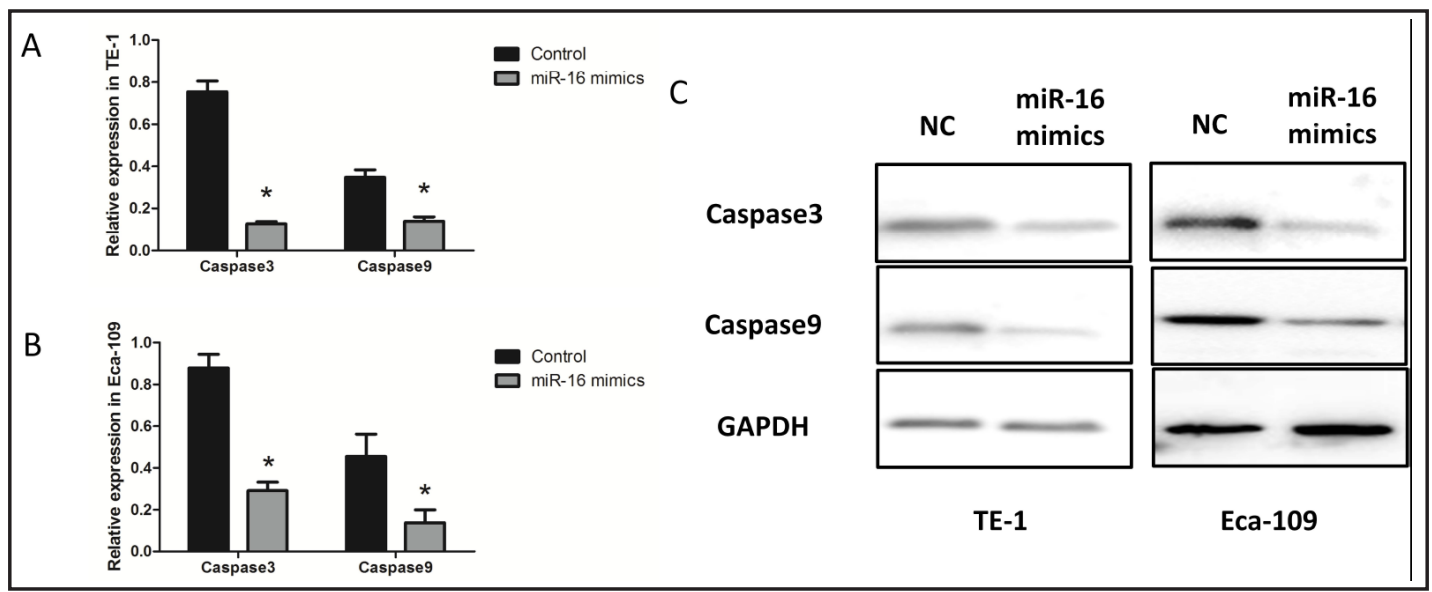

Fig. 5. A, B: The expression level of the mRNA of Caspase 3 and 9 were detected by RT-PCR in cells treated with miR-16 and control. C: The protein of the cleaved Caspase 3 and 9 were examined by western blot. * indicates $\mathrm{P}<0.05$. All tests were performed in triplicate and presented as mean $\pm \mathrm{SE}$.

Fig. 6. The mRNA levels of RECK and SOX6 n human ESCC tissues and adjacent corresponding tissues $(n=40)$ were evaluated by qRT-PCR. A, B.:Data were presented as box plot of the median and range of log-transformed relative expression level. * indicates $\mathrm{P}<0.05$. The protein expression level of RECK and SOX6 were detected by western blot. C: Remarkable decreased level of RECK and SOX6 in both mRNA and protein level was detected in ESCC tissues.

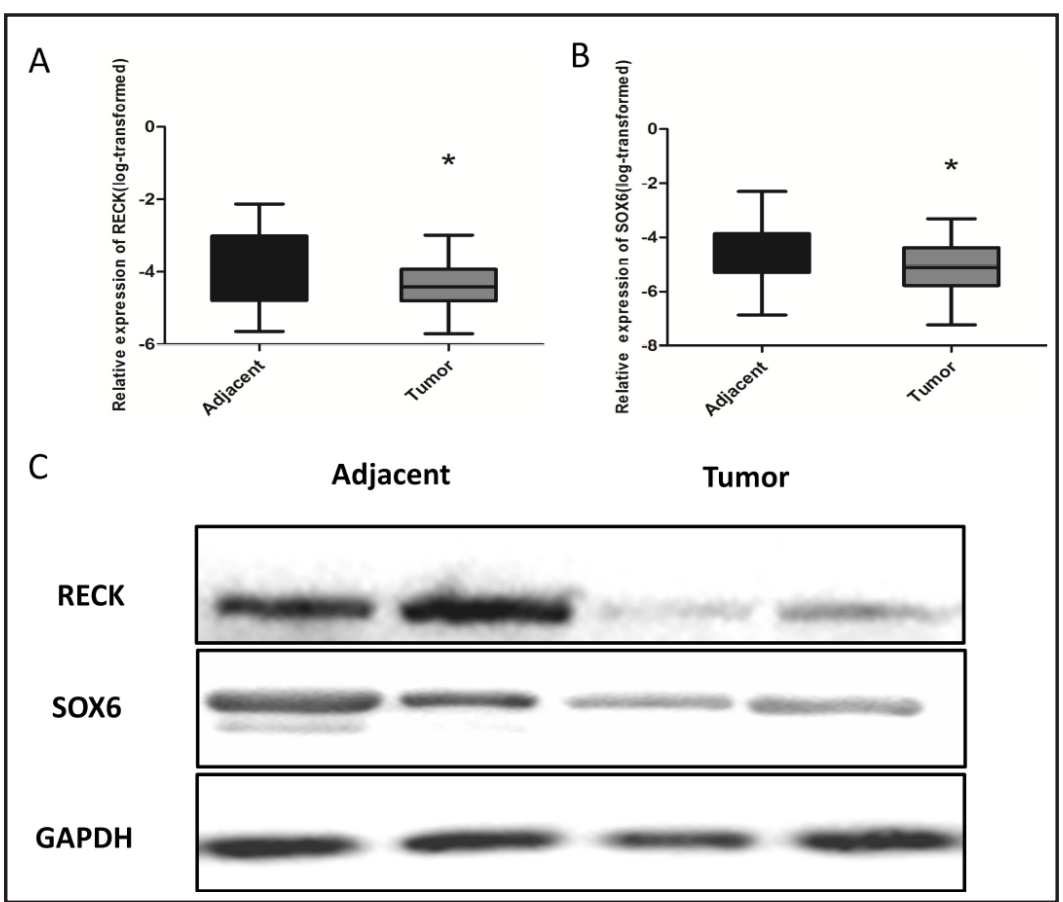

RECK and SOX6 was the direct targets of miR-16

Since miR-16 could suppress the cell apoptosis in ESCC cell lines, we were confusing about the potential mechanism in which miR-16 could affect cell apoptosis. Based on the regulation pattern of miRNAs in human carcinoma, we collected the candidate genes which have been reported associating with cell apoptosis as well as regulated by miRNAs. Furthermore, we predicated the potential target genes of miR-16 by using bioinformatics software. Finally, we selected the 5 overlap genes by using the two approaching above including RECK, CCNE1, FGF2, SOX6, BCL2L2.

RT-PCR was conducted to screen the candidate genes we selected above in 40 pairs ESCC tissues. The results indicated that only the RECK and SOX6 were aberrant down-regulated in tumor tissues when comparing with the adjacent tissues. Thus, we confirmed the protein expression levels of the two genes in these tissues. We found that the protein expression level was similarly decreased (Fig. 6). 


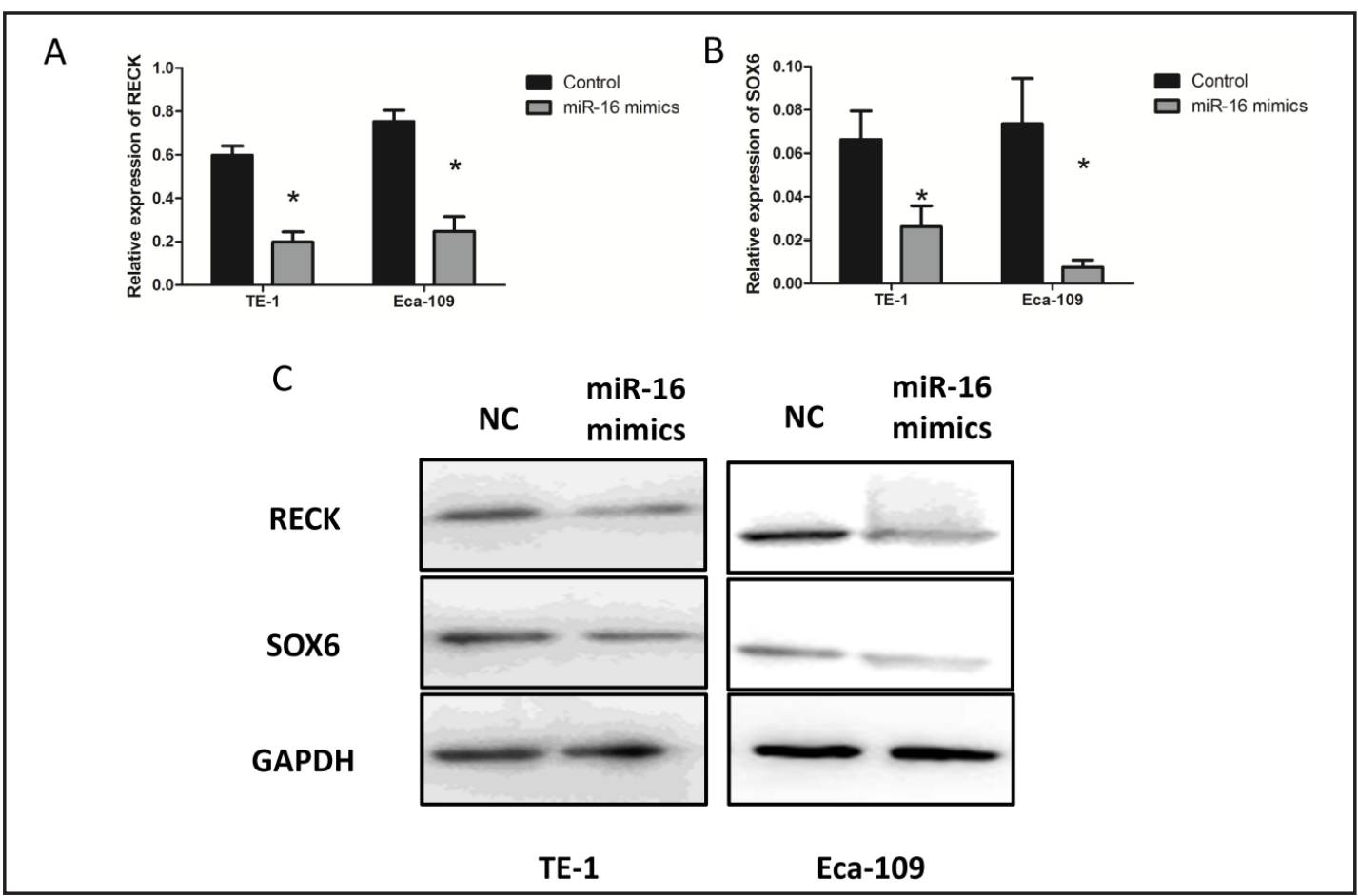

Fig. 7. Cells were transfected with $50 \mathrm{nM}$ miR-16 mimics for $48 \mathrm{~h}$. qRT-PCR was performed to evaluate the mRNA level of RECK and SOX6 (A, B). RECK and SOX6 protein expression levels were analyzed by westernblotting $(C)$. * indicates significant difference $(\mathrm{P}<0.05)$.

To further detected the abnormal expression level of RECK and SOX6 was induced by miR-16, we examined the level of the two genes in cells transfected with miR-16 mimics and control. Remarkable suppression level of the two genes was obtained in cells transfected with miR-16 which indicated that miR-16 may negatively regulate RECK and SOX6 in ESCC cell lines (Fig. 7).

Whether the suppression level of RECK and SOX6 induced by miR-16 was due to the binding of miR-16 in the 3'UTR of the two genes still remain unknown. Next, we performed miRNA luciferase reporter assay by constructing the wild type and mutant type luciferase reporter plasmids containing the binding region of the 3'UTR of RECK or SOX6 mRNAs. We found that co-transfection of miR-16 mimics and pGL3-RECK or pGL3-SOX6 3'UTR reporter plasmids significantly decreased the luciferase activity in cell lines, as compared with the control and the mutant type, suggesting miR-16 directly targets both RECK and SOX6 (Fig. 8).

\section{Discussion}

Researchers have found that miR-16 could decrease the level of CCND1 in osteosarcoma which induced apoptosis and cell cycle arrest [13]. Besides, Bcl2, another target gene of miR-16, was involved in the activation of pancreatic stellate cells [14]. By binding the 3'UTR region of the multiple target genes, miR-16 could affect the apoptosis in various diseases.

RECK, as one of the target genes of miR-16; suppress tumor invasion and metastasis by negatively regulating matrix metalloproteinase 9 (MMP9) activity. The expression of RECK was closely correlated with histological grade, infiltrative depth and lymphatic metastasis in ESCC [15]. Our study indicate that RECK is targets of miR-16, and miR-16 over-expression can inhibit the apoptosis of ESCC cells. Western blot assay in our study showed that RECK 


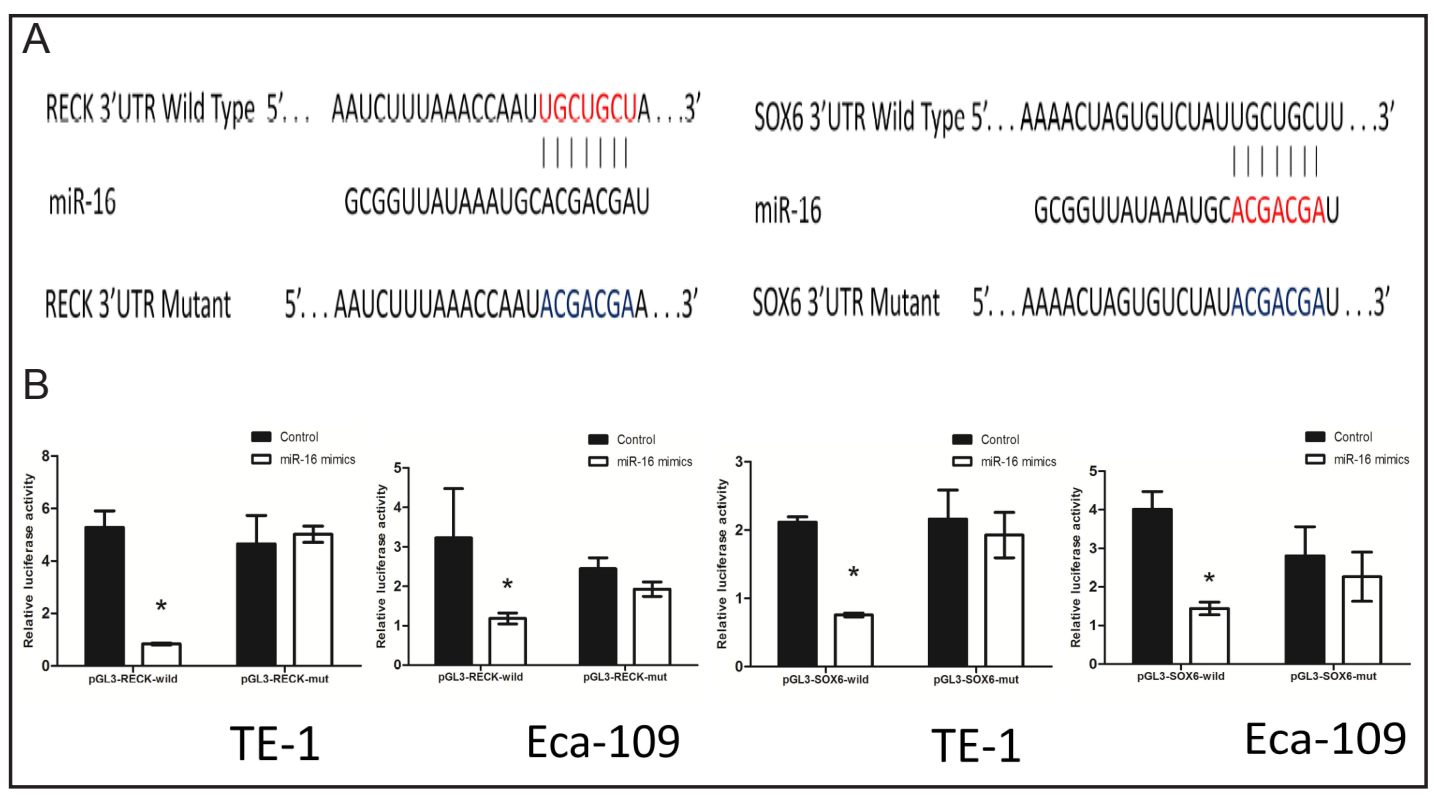

Fig. 8. A: Sequence alignment of human miR-16 with 3' UTR of RECK and SOX6. Bottom: mutations in the 3'-UTR of RECK and SOX6 in order to create the mutant luciferase reporter construct. B: Cells were cotransfected with miR-16 mimics or miR-control, renilla luciferase vector pGL3-SV40 and RECK or SOX6 3'UTR luciferase reporters for $48 \mathrm{~h}$. Firefly luciferase signals were normalized with Renilla luciferase signals. * indicates $\mathrm{P}<0.05$.

expression of cell lines were decreased significantly after the cells were treated with miR-16 mimics, and the luciferase reporter assay also showed the same result. So, we hypothesize that miR-16 can inhibit apoptosis ability by targeting RECK. Our experimental results help us understand the molecular basis of miR-16 and give clues for potential therapeutic methods for ESCC.

SOX6 was found frequently down-regulated in primary ESCCs in both mRNA level and protein level, which was significantly associated with the poor differentiation, lymph node metastases, advanced TNM stage and disease-specific survival, indicating that SOX6 played a crucial role in the pathogenesis of ESCC [16]. SOX6, a member of the D subfamily of sex determining region y-related transcription factors, plays critical roles in cell fate determination, differentiation and proliferation. It has been identified as a tumor suppressor or an oncogene in different human cancers [17]. We observed that both SOX6 mRNA and protein levels were significantly decreased in tumor tissues compared with adjacent corresponding tissues. In addition, the SOX6 protein and mRNA showed similar expression patterns in the matched samples. Consistent with the observation of Qin et al. on esophageal squamous cell carcinoma [16], these findings support the hypothesis that SOX6 may play an important role in the tumor-genesis of ESCC. It has been reported that It has been reported that SOX6 inhibits cyclin D1 expression by interacting with $\beta$-catenin and HDAC1 in insulinoma INS-1E and NIH-3T3 cells [18]. Furthermore, Sox6 play an important role in the turning-point at which P19 cells stimulated with RA proceed to cell death or differentiate which indicated that the SOX6 induced apoptosis.

The aberrant expression level of miR-16 in ESCC tissues was high associated with tumor size. According to our results, over-expressed level of miR-16 induced the inhibition of cell apoptosis, thus, we assumed that the loss of cell apoptosis could result in the proliferation of tumor cell. In conclusion, miR-16 may affect the proliferation and apoptosis of ESCC cells by down-regulated RECK and SOX6, playing an important role in the pathogenesis of ESCC. The important role of miR-16 might provide a foundation of a new diagnosis method or a novel targeted therapy for ESCC. 


\begin{tabular}{|c|c|c|}
\hline Cellular Physiology & Cell Physiol Biochem 2014;33:1340-1348 & \\
\hline and Biochemistry & $\begin{array}{l}\text { DOI: 10.1159/000358701 } \\
\text { Publisned online: May 05, } 2014\end{array}$ & $\begin{array}{l}\text { O } 2014 \text { S. Karger AG, Basel } \\
\text { www.karger.com/cpb }\end{array}$ \\
\hline
\end{tabular}

\section{References}

1 Shigaki H, Baba Y, Watanabe M, Murata A, Ishimoto T, Iwatsuki M, Iwagami S, Nosho K, Baba H: Pik3ca mutation is associated with a favorable prognosis among patients with curatively resected esophageal squamous cell carcinoma. Clin Cancer Res 2013;19:2451-2459.

-2 Zhu YH, Fu L, Chen L, Qin YR, Liu H, Xie F, Zeng T, Dong SS, Li J, Li Y, Dai Y, Xie D, Guan XY: Downregulation of the novel tumor suppressor diras1 predicts poor prognosis in esophageal squamous cell carcinoma. Cancer Res 2013;73:2298-2309.

- 3 Tang KH, Dai YD, Tong M, Chan YP, Kwan PS, Fu L, Qin YR, Tsao SW, Lung HL, Lung ML, Tong DK, Law S, Chan KW, Ma S, Guan XY: A cd90(+) tumor-initiating cell population with an aggressive signature and metastatic capacity in esophageal cancer. Cancer Res 2013;73:2322-2332.

- Kong KL, Kwong DL, Chan TH, Law SY, Chen L, Li Y, Qin YR, Guan XY: Microrna-375 inhibits tumour growth and metastasis in oesophageal squamous cell carcinoma through repressing insulin-like growth factor 1 receptor. Gut 2012;61:33-42.

5 Gong H, Song L, Lin C, Liu A, Lin X, Wu J, Li M, Li J: Downregulation of mir-138 sustains nf-kappab activation and promotes lipid raft formation in esophageal squamous cell carcinoma. Clin Cancer Res 2013;19:1083-1093.

-6 Asangani IA, Rasheed SA, Nikolova DA, Leupold JH, Colburn NH, Post S, Allgayer H: Microrna-21 (mir-21) post-transcriptionally downregulates tumor suppressor pdcd 4 and stimulates invasion, intravasation and metastasis in colorectal cancer. Oncogene 2008;27:2128-2136.

7 Simon DJ, Madison JM, Conery AL, Thompson-Peer KL, Soskis M, Ruvkun GB, Kaplan JM, Kim JK: The microrna mir-1 regulates a mef-2-dependent retrograde signal at neuromuscular junctions. Cell 2008;133:903-915.

8 Kimura S, Naganuma S, Susuki D, Hirono Y, Yamaguchi A, Fujieda S, Sano K, Itoh H: Expression of micrornas in squamous cell carcinoma of human head and neck and the esophagus: Mir-205 and mir-21 are specific markers for hnscc and escc. Oncol Rep 2010;23:1625-1633.

-9 Ogawa R, Ishiguro H, Kuwabara Y, Kimura M, Mitsui A, Katada T, Harata K, Tanaka T, Fujii Y: Expression profiling of micro-rnas in human esophageal squamous cell carcinoma using rt-pcr. Med Mol Morphol 2009;42:102-109.

10 Jiang QQ Liu B, Yuan T: Microrna-16 inhibits bladder cancer proliferation by targeting cyclin d1. Asian Pac J Cancer Prev 2013;14:4127-4130.

11 Ke Y, Zhao W, Xiong J, Cao R: Downregulation of mir-16 promotes growth and motility by targeting hdgf in non-small cell lung cancer cells. FEBS Lett 2013;587:3153-3157.

-12 Ying H, Lv J, Ying T, Li J, Yang Q, Ma Y: Microrna and transcription factor mediated regulatory network for ovarian cancer: Regulatory network of ovarian cancer. Tumour Biol 2013;34:3219-3225.

13 Cai CK, Zhao GY, Tian LY, Liu L, Yan K, Ma YL, Ji ZW, Li XX, Han K, Gao J, Qiu XC, Fan QY, Yang TT, Ma BA: Mir15a and mir-16-1 downregulate ccnd1 and induce apoptosis and cell cycle arrest in osteosarcoma. Oncol Rep 2012;28:1764-1770.

14 Shen J, Wan R, Hu G, Yang L, Xiong J, Wang F, He S, Guo X, Ni J, Guo C, Wang X: Mir-15b and mir-16 induce the apoptosis of rat activated pancreatic stellate cells by targeting bcl-2 in vitro. Pancreatology 2012;12:91-99.

15 Wang L, Wang Q, Li HL, Han LY: Expression of mir200a, mir93, metastasis-related gene reck and mmp2/ mmp9 in human cervical carcinoma--relationship with prognosis. Asian Pac J Cancer Prev 2013;14:21132118.

16 Qin YR, Tang H, Xie F, Liu H, Zhu Y, Ai J, Chen L, Li Y, Kwong DL, Fu L, Guan XY: Characterization of tumorsuppressive function of sox6 in human esophageal squamous cell carcinoma. Clin Cancer Res 2011;17:4655.

17 Wagner T, Wirth J, Meyer J, Zabel B, Held M, Zimmer J, Pasantes J, Bricarelli FD, Keutel J, Hustert E, Wolf U, Tommerup N, Schempp W, Scherer G: Autosomal sex reversal and campomelic dysplasia are caused by mutations in and around the sry-related gene sox9. Cell 1994;79:1111-1120.

18 Iguchi H, Urashima Y, Inagaki Y, Ikeda Y, Okamura M, Tanaka T, Uchida A, Yamamoto TT, Kodama T, Sakai J: Sox6 suppresses cyclin d1 promoter activity by interacting with beta-catenin and histone deacetylase 1, and its down-regulation induces pancreatic beta-cell proliferation. J Biol Chem 2007;282:19052-19061. 